\title{
Synchronous and asynchronous boundary temperature modulations of Bénard-Darcy convection
}

\author{
P.G. Siddheshwar ${ }^{\mathrm{a}, *}$, B.S. Bhadauria ${ }^{\mathrm{b}}$, Om P. Suthar ${ }^{\mathrm{a}}$ \\ ${ }^{a}$ Department of Mathematics, Bangalore University, Central College Campus, Bangalore 560001 , India \\ ${ }^{\mathrm{b}}$ Department of Applied Mathematics, Babasaheb Bhimrao Ambedkar University, Lucknow 226 025, India
}

\section{A R T I C L E I N F O}

\section{Article history:}

Received 1 June 2012

Received in revised form

11 September 2012

Accepted 11 September 2012

Available online 26 September 2012

Keywords:

Bénard-Darcy convection

Weakly non-linear stability analysis

Ginzburg-Landau amplitude equation

Temperature modulation

Nusselt number

\begin{abstract}
A B S T R A C T
A theoretical analysis of thermo-convective instability in a densely packed porous medium is carried out when the boundary temperatures vary with time in a sinusoidal manner. By performing a weakly non-linear stability analysis, the Nusselt number is obtained as a function of amplitude of convection which is governed by a non-autonomous Ginzburg-Landau equation derived for the stationary mode of convection. The paper succeeds in unifying the modulated Bénard-Darcy, Bénard-Rayleigh, BénardBrinkman and Bénard-Chandrasekhar convection problems and hence precludes the study of these individual problems in isolation. A new result that shows that asynchronous temperature modulation may be effectively used to either enhance or reduce heat transport by suitably adjusting the frequency and phase-difference of the modulated temperature is presented.
\end{abstract}

(c) 2012 Elsevier Ltd. All rights reserved.

\section{Introduction}

Bénard-Rayleigh convection and its porous medium analog, namely, Bénard-Darcy convection are well-explored problems that now find mention in excellent books (see [7,19,11,14,17,32,31]). In these two celebrated problems the quiescent basic state temperature is time-independent due to the application of uniform temperature at the boundaries. When time-dependent temperatures are imposed on the boundaries, in a synchronous or on asynchronous manner, then the governing equations of both linear and non-linear stability have time-periodic coefficients. The work of Venezian [33], Rosenblat and Herbert [25], Rosenblat and Tanaka [24], Davis [9], Homsy [13], Roppo et al. [23], Ahlers et al. [1], Niemmela and Donnelly [18], Bhadauria and Bhatia [5], Bhadauria et al. [4], and Raju and Bhattacharya [21] are some of the reported works that concern the onset of the thermallymodulated Bénard-Rayleigh convection. The analogous onset problem in porous media has also been investigated by many researchers (see $[8,16,3,2]$ and references therein). In addition, there are papers that address the thermally modulated Bénard-Chandrasekhar problem (see [30]) in electrically conducting liquids. Restricting attention to problems involving Newtonian liquids, it is to be noted that the thermally modulated Bénard-Rayleigh, Bénard-Darcy, Bénard-Brinkman, and Bénard-Chandrasekhar convection problems are reported in the literature as independent problems since they were

\footnotetext{
* Corresponding author. Tel.: +918022961432.

E-mail address: mathdrpgs@gmail.com (P.G. Siddheshwar).
}

investigated at a time when connection between them seems to have been not recognized. Even to this day many works get reported on these problems in isolation. In the context of the above observations the objectives set down in the paper are:

- Present the linear matrix differential operator method to find the Ginzburg-Landau amplitude equation for the modulated problem.

- Use the solution of the Ginzburg-Landau equation in quantifying the amount of heat transport.

- Unify the four thermally modulated problems of Bénard-Rayleigh, Bénard-Darcy, Bénard-Brinkman, and Bénard-Chandrasekhar.

- Find the condition under which the local acceleration term can be neglected in the modulation problem.

- Present some new results on the Nusselt number whose maximum and minimum values depend crucially on the choice of frequency and phase-difference, rather than on frequency alone.

- Explore the possibility of regulating heat transport by a mechanism that is external to the convective system.

The formulation of the modulated Bénard-Darcy system that has lower order governing equations than Bénard-Rayleigh, BénardBrinkman and Bénard-Chandrasekhar systems now follows.

\section{Problem formulation}

An infinite extent horizontal porous layer of thickness $d$, whose lower and upper bounding planes are at $z=0$ and $z=d$ 
respectively, is considered (Fig. 1). The porous layer is saturated by a viscous, Newtonian liquid that is heated from below and cooled from above in a time-periodic manner. Further, the porous medium is supposed to be anisotropic, and Darcy law and the Oberbeck-Boussinesq approximation [20] are taken to be applicable. The other assumptions such as local thermal equilibrium, no heating from viscous dissipation and no radiative effects are also assumed. The equations which describe this system are [17] the following.

Conservation of mass

$\nabla \cdot \vec{q}=0$.

Conservation of momentum

$\frac{1}{\phi} \frac{\partial \vec{q}}{\partial t}=-\frac{1}{\rho_{0}} \nabla p+\frac{\rho}{\rho_{0}} \vec{g}-v \underline{K} \cdot \vec{q}$.

Conservation of energy

$\left.\frac{\partial T}{\partial t}+\frac{1}{\phi}(\vec{q} \cdot \nabla) T=\nabla \cdot \underline{\left(\kappa_{e}\right.} \cdot \nabla T\right)$.

Equation of state

$\rho=\rho_{0}\left[1-\beta\left(T-T_{0}\right)\right]$,

where $\vec{q}$ is the velocity, $\phi$ is the porosity of the porous medium, $\rho$ is the density of the fluid, $p$ is hydrodynamic pressure, $\vec{g}=$ $(0,0,-g)$ is the gravitational force, $v$ is the kinematic viscosity, $T$ is the temperature, $\underline{K}$ and $\underline{\kappa_{e}}$ are the permeability and thermal diffusivity tensors, given by

$\underline{K}=\frac{1}{K_{v}}\left[\begin{array}{ccc}\frac{1}{\xi} & 0 & 0 \\ 0 & \frac{1}{\xi} & 0 \\ 0 & 0 & 1\end{array}\right], \quad \underline{\kappa_{e}}=\underline{\kappa_{v}}\left[\begin{array}{ccc}\eta & 0 & 0 \\ 0 & \eta & 0 \\ 0 & 0 & 1\end{array}\right]$,

where $\xi=K_{h} / K_{v}$ and $\eta=\kappa_{h} / \kappa_{v}$ are the mechanical and thermal anisotropy parameters.

The externally imposed time-periodic boundary temperatures are assumed in the following form [33]:

$$
\begin{aligned}
T & =T_{0}+\frac{\Delta T}{2}[1+\epsilon \cos (\omega t)] \text { at } z=0, \\
& =T_{0}-\frac{\Delta T}{2}[1-\epsilon \cos (\omega t+\theta)] \text { at } z=d,
\end{aligned}
$$

where $\epsilon$ is the small amplitude of modulation, $\omega$ is the modulation frequency and $\theta$ is the phase difference.

The basic state is assumed to be quiescent and the quantities in this state are given by

$\overrightarrow{q_{b}}=(0,0,0), \quad \rho=\rho_{b}(z, t), \quad p=p_{b}(z, t), \quad T=T_{b}(z, t)$.

These basic state quantities satisfy the following equations:

$\frac{\partial p_{b}}{\partial z}=-\rho_{b} g$

$\frac{\partial T_{b}}{\partial t}=\kappa_{v} \frac{\partial^{2} T_{b}}{\partial z^{2}}$

$\rho_{b}=\rho_{0}\left[1-\beta\left(T_{b}-T_{0}\right)\right]$.

The solution of Eq. (7), subject to the boundary conditions (5), is given by

$T_{b}(z, t)=T_{0}+\Delta T\left(1-\frac{z}{d}\right)+\epsilon F(z, t)$,

where

$F(z, t)=\frac{1}{2}[f(z) \exp (i \omega t)+\tilde{f}(z) \exp (-i \omega t)]$,

$f(z)=A(\lambda) e^{\lambda z / d}+A(-\lambda) e^{-\lambda z / d}$,
$A(\lambda)=\frac{1}{2} \frac{\left(e^{-i \theta}-e^{-\lambda}\right)}{\left(e^{\lambda}-e^{-\lambda}\right)}, \quad \lambda=(1-i) \sqrt{\frac{\omega}{2}}$,

and a tilde over a quantity indicates its conjugate. The perturbations on the basic state are superposed in the form

$\vec{q}=\vec{q}_{b}+\vec{q}^{\prime} ; \quad p=p_{b}+p^{\prime} ; \quad T=T_{b}+T^{\prime} ; \quad \rho=\rho_{b}+\rho^{\prime}$,

where the perturbations are of finite amplitude.

Substituting Eq. (13) in Eqs. (1)-(4) and using Eq. (9) in the resulting equations, the following equations are arrived at

$\nabla \cdot \overrightarrow{q^{\prime}}=0$

$\frac{1}{\phi} \frac{\partial \vec{q}^{\prime}}{\partial t}=-\frac{1}{\rho_{0}} \nabla p^{\prime}+\frac{\rho}{\rho_{0}} \vec{g}-v \underline{K} \cdot \overrightarrow{q^{\prime}}$,

$\frac{\partial T^{\prime}}{\partial t}+\frac{1}{\phi}\left(\overrightarrow{q^{\prime}} \cdot \nabla\right) T^{\prime}+w^{\prime} \frac{\partial T_{b}}{\partial z}=\nabla \cdot\left(\underline{\kappa_{e}} \cdot \nabla T^{\prime}\right)$,

$\rho^{\prime}=-\rho_{0} \beta T^{\prime}$

Only two-dimensional disturbances are considered, i.e. $v^{\prime}=0$, and we define the stream function $\psi^{\prime}$ as

$u^{\prime}=\frac{\partial \psi^{\prime}}{\partial z}, \quad w^{\prime}=-\frac{\partial \psi^{\prime}}{\partial x}$.

Eliminating the pressure term from Eq. (15) and then introducing the stream function the following equation is obtained:

$\frac{1}{\phi} \frac{\partial}{\partial t}\left(\nabla^{2} \psi^{\prime}\right)=-\frac{v}{K_{v}} \nabla_{\xi}^{2} \psi^{\prime}-\beta g \frac{\partial T^{\prime}}{\partial x}$.

On substituting Eq. (18) in Eq. (16), the heat transport equation is got in the form

$\frac{\partial T^{\prime}}{\partial t}-\frac{1}{\phi} \frac{\partial\left(\psi^{\prime}, T^{\prime}\right)}{\partial(x, z)}=\kappa_{v} \nabla_{\eta}^{2} T^{\prime}+\frac{1}{\phi} \frac{\partial \psi^{\prime}}{\partial x} \frac{\partial T_{b}}{\partial z}$,

where

$\nabla^{2} \equiv \frac{\partial^{2}}{\partial x^{2}}+\frac{\partial^{2}}{\partial z^{2}}, \quad \nabla_{\xi}^{2} \equiv \frac{\partial^{2}}{\partial x^{2}}+\frac{1}{\xi} \frac{\partial^{2}}{\partial z^{2}}, \quad \nabla_{\eta}^{2} \equiv \eta \frac{\partial^{2}}{\partial x^{2}}+\frac{\partial^{2}}{\partial z^{2}}$.

Eqs. (18)-(19) are now rendered dimensionless using the following scaling:

time $\rightarrow d^{2} / \kappa_{v}$, space-coordinates $\rightarrow d$,

temperature $\rightarrow \Delta T$, streamfunction $\rightarrow \phi \kappa_{v}$.

The dimensionless form of Eqs. (18)-(19) are

$\frac{1}{V a} \frac{\partial}{\partial t_{*}}\left(\nabla_{*}^{2} \psi_{*}^{\prime}\right)=-\nabla_{\xi *}^{2} \psi_{*}^{\prime}-R a \frac{\partial T_{*}^{\prime}}{\partial x_{*}}$,

$\frac{\partial T_{*}^{\prime}}{\partial t_{*}}-\frac{\partial\left(\psi_{*}^{\prime}, T_{*}^{\prime}\right)}{\partial\left(x_{*}, Z_{*}\right)}=\nabla_{\eta}^{2} T_{*}^{\prime}+\frac{\partial \psi_{*}^{\prime}}{\partial x_{*}} \frac{\partial T_{b *}}{\partial z_{*}}$,

where asterisks denote dimensionless values. In the above equations, the dimensionless parameters are Vadasz number $(V a)$ and Darcy-Rayleigh number $(R a)$ that are respectively given by

$V a=\frac{\phi v d^{2}}{K_{v} \kappa_{v}}, \quad R a=\frac{\beta \Delta T g d K_{v}}{\phi v \kappa_{v}}$

The boundary conditions for the perturbed quantities are

$\psi_{*}^{\prime}=0 \quad$ at $z_{*}=0,1$

$T_{*}^{\prime}=0 \quad$ at $\left.z_{*}=0,1\right\}$.

For the sake of convenience, the asterisks are dropped in what follows. It is to be noted that the basic state solution influences the stability problem through the factor $\partial T_{b} / \partial z$ in Eq. (22), which is given by

$\frac{\partial T_{b}}{\partial z}(z, t)=-1+\frac{\epsilon}{2}\left[f_{1}(z) \exp (i \omega t)+\tilde{f_{1}}(z) \exp (-i \omega t)\right]$, 
where

$f_{1}(z)=A_{1}(\lambda) e^{\lambda z}+A_{1}(-\lambda) e^{-\lambda z}$,

$A_{1}(\lambda)=\frac{\lambda}{2} \frac{\left(e^{-i \theta}-e^{-\lambda}\right)}{\left(e^{\lambda}-e^{-\lambda}\right)}, \quad \lambda=(1-i) \sqrt{\frac{\omega}{2}}$.

The critical Rayleigh number for the unmodulated case is given by Epherre [10]

$\Psi_{s}^{(0)}=A \sin (\pi z), \quad R a_{c}^{(0)}=\frac{\alpha_{1}^{2} \alpha_{2}^{2}}{k_{c}^{2}}$,

where $\alpha^{2}=k_{c}^{2}+\pi^{2}, \alpha_{1}^{2}=k_{c}^{2}+\xi^{-1} \pi^{2}$ and $\alpha_{2}^{2}=\eta k_{c}^{2}+\pi^{2}$.

It can easily be shown that $k_{c}=\pi /\left((\eta \xi)^{1 / 4}\right)$ and hence

$R a_{c}^{(0)}=\left[1+\sqrt{\frac{\eta}{\xi}}\right]^{2} \pi^{2}$

These are the critical values of wave number and Rayleigh number of the unmodulated Bénard-Darcy anisotropic system [10].

Having formulated the thermally modulated Bénard-Darcy system, the heat transport through the lower boundary will be quantified next. The linear matrix differential operator method will be used in what follows to transform the non-linear stability equations into a system of inhomogeneous linear matrix differential equations. The aim of the next section is to achieve the objectives mentioned in the Introduction.

\section{Weakly non-linear stability analysis}

Using the time variations at only the slow time scale, viz., $\tau=\delta^{2} t$ where $\delta$ is a small quantity, Eqs. (20)-(21) now take the form

$$
\left[\begin{array}{cc}
\nabla_{\xi}^{2} & R a \frac{\partial}{\partial x} \\
\frac{\partial}{\partial x} & -\nabla_{\eta}^{2}
\end{array}\right]\left[\begin{array}{c}
\psi^{\prime} \\
T^{\prime}
\end{array}\right]=\left[\begin{array}{c}
-\frac{\delta^{2}}{V a} \frac{\partial \psi^{\prime}}{\partial \tau} \nabla^{2} \\
-\delta^{2} \frac{\partial T^{\prime}}{\partial \tau}+\frac{\partial\left(\psi^{\prime}, T^{\prime}\right)}{\partial(x, z)}+\delta^{2} \epsilon_{2} \frac{\partial F}{\partial z}
\end{array}\right]
$$

In writing Eq. (27) it has been assumed that the modulation is of small amplitude and hence $\epsilon=\delta^{2} \epsilon_{2}$. This is in keeping with the assumption of Govender [12] and Siddheshwar et al. [28,29]. With the aim of deriving the Ginzburg-Landau equation for the amplitude of convection, we now use the following asymptotic

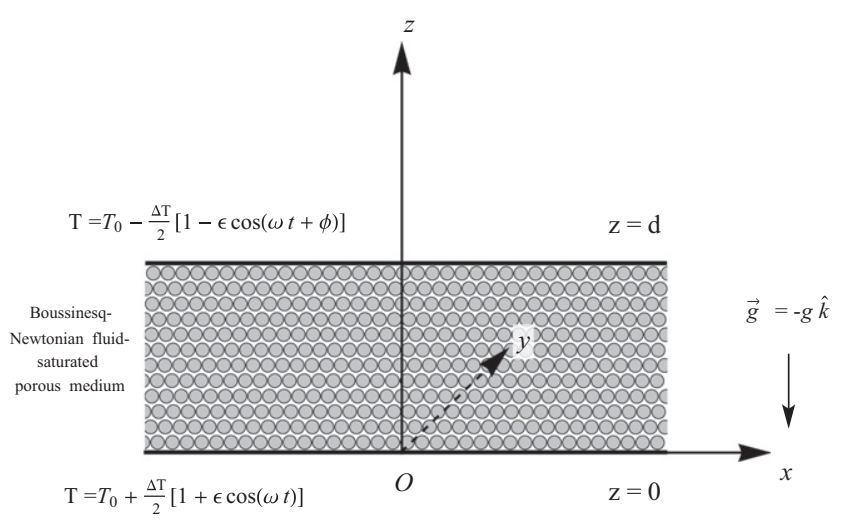

Fig. 1. Schematic diagram of the problem. (For interpretation of the references to color in this figure caption, the reader is referred to the web version of this article.) expansion in Eq. (27) [6]

$$
\left.\begin{array}{l}
R a=R a_{0}+\delta^{2} R a_{2}+\cdots \\
\psi^{\prime}(x, z, \tau)=\delta \psi_{1}(x, z, \tau)+\delta^{2} \psi_{2}(z, \tau)+\cdots \\
T^{\prime}(x, z, \tau)=\delta \Theta_{1}(x, z, \tau)+\delta^{2} \Theta_{2}(z, \tau)+\cdots
\end{array}\right\},
$$

where $R a_{0}$ is the critical value of the Rayleigh number at which Bénard-Darcy convection sets in when modulation is absent. Following Siddheshwar et al. [27], the first- and second-order solutions may be shown to be the following:

$$
\left.\begin{array}{ll}
\psi_{1}=\mathcal{A}(\tau) \sin \left(k_{c} x\right) \sin (\pi z), & \Theta_{1}=-\frac{k_{c}}{\alpha_{2}^{2}} \mathcal{A}(\tau) \cos \left(k_{c} x\right) \sin (\pi z) \\
\psi_{2}=0, & \Theta_{2}=-\frac{k_{c}^{2}}{8 \pi \alpha_{2}^{2}} \mathcal{A}^{2}(\tau) \sin (2 \pi z)
\end{array}\right\} .
$$

Using the solutions of the first- and second-order systems, the third-order system takes the form

$\left[\begin{array}{cc}\nabla_{\xi}^{2} & R a_{0} \frac{\partial}{\partial x} \\ \frac{\partial}{\partial x} & -\nabla_{\eta}^{2}\end{array}\right]\left[\begin{array}{l}\psi_{3} \\ \Theta_{3}\end{array}\right]=\left[\begin{array}{l}\mathcal{R}_{31} \\ \mathcal{R}_{32}\end{array}\right]$

where

$\mathcal{R}_{31}=\left(\frac{\alpha^{2}}{V a} \frac{d \mathcal{A}}{d \tau}-R_{2} \frac{k_{c}^{2}}{\alpha_{2}^{2}} \mathcal{A}\right) \sin \left(k_{c} x\right) \sin (\pi z)$,

$\mathcal{R}_{32}=\left(\frac{k_{c}}{\alpha_{2}^{2}} \frac{d \mathcal{A}}{d \tau}+\epsilon_{2} \frac{\partial F}{\partial z} k_{c} \mathcal{A}-\frac{k_{c}^{3}}{4 \alpha_{2}^{2}} \mathcal{A}^{3} \sin (2 \pi z)\right) \cos \left(k_{c} x\right) \sin (\pi z)$.

The solvability condition [26] for the existence of the third-order solution yields the Ginzburg-Landau equation for stationary instability with a time-periodic coefficient in the form

$$
\begin{aligned}
& \frac{1}{\alpha_{2}^{2}}\left(1+\frac{\alpha^{2} \alpha_{2}^{2}}{\alpha_{1}^{2} V a}\right) \frac{d \mathcal{A}(\tau)}{d \tau}-\left[\frac{R a_{2}}{R a_{0}}-2 \epsilon_{2} \mathcal{L}(\tau)\right] \mathcal{A}(\tau) \\
& \quad+\frac{k_{c}^{2}}{8 \alpha_{2}^{2}} \mathcal{A}^{3}(\tau)=0
\end{aligned}
$$

where

$\mathcal{L}(\tau)=\int_{0}^{1}\left[\frac{\partial F(z, \tau)}{\partial z} \sin (\pi z)\right] d z$

As Eq. (32) is non-autonomous, we numerically integrate the equation using NDSolve of Mathematica 8.0 with the initial condition $\mathcal{A}(0)=a_{0}$. Without loss of generality $R a_{2}=R a_{0}$ is assumed in the calculations and this is done to keep the parameters to a minimum. From Eq. (28), we have for $R a_{2}=R a_{0}$

$\frac{R a}{1+\delta^{2}}=R a_{0}$

This essentially means that the actual Rayleigh number is diminished as a result of this assumption. In the event of $R a_{2}$ becoming negative it would mean that the actual Rayleigh number is enhanced.

The heat transport through the lower boundary is now quantified in the form of the horizontally-averaged Nusselt number, $\mathrm{Nu}$, defined by

$N u(\tau)=1+\frac{\left[\frac{k_{c}}{2 \pi} \int_{0}^{2 \pi / k_{c}} \frac{d \Theta_{2}}{d z} d x\right]_{z=0}}{\left[\frac{k_{c}}{2 \pi} \int_{0}^{2 \pi / k_{c}} \frac{\partial T_{b}}{\partial z} d x\right]_{z=0}}$

Substituting $\Theta_{2}$ and $\partial T_{b} / \partial z$ respectively from Eqs. (28) and (23) into Eq. (34) and completing the integration, the expression for 
$\mathrm{Nu}(\tau)$ reads as follows:

$N u(\tau)=1+\frac{k_{c}^{2}}{4 \alpha_{2}^{2}} A^{2}(\tau)$.

To have a good understanding of the effect of thermal modulation on heat transport a representative time interval that allows a clear comprehension of the modulation effect needs to be chosen. The interval $[0,2 \pi]$ seemed an appropriate interval to calculate a mean Nusselt number and this is decided upon by careful examination of the results in Figs. 2-3. The time-averaged Nusselt number $\overline{N u}$ is thus defined as

$\overline{N u}=\frac{1}{2 \pi} \int_{0}^{2 \pi} N u(\tau) d \tau$.

The amplitude $\mathcal{A}(\tau)$ is obtained numerically and hence $\overline{N u}$ is also to be numerically evaluated. An interesting observation that can be made per se by having a cursory glance at Eq. (32) is that the sign of $\mathcal{L}(\tau)$ determines whether the modulation amplifies or diminishes the amplitude of convection. In other words depending on the need of an application $\mathcal{L}(\tau)$, which represents the thermal modulation effect, may be manipulated upon to either facilitate enhancement or reduction in heat transport. A discussion of the results now follows culminating in a listing of conclusions.

\section{Results and discussion}

The paper is an attempt to bring in the individual effect of synchronous and asynchronous temperature modulations on the onset of Bénard-Darcy convection as well as on heat transport in a non-linear realm of convection. The following two types of modulations are considered:

1. Synchronous temperature modulation, in which case $\theta=0$.

2. Asynchronous temperature modulation, in which case $\theta \neq 0$.

The porous medium is assumed to be closely packed, and hence the use of the Darcy model in the governing equations. In addition, the term $V a^{-1} \partial / \partial t$ has been brought into the model to
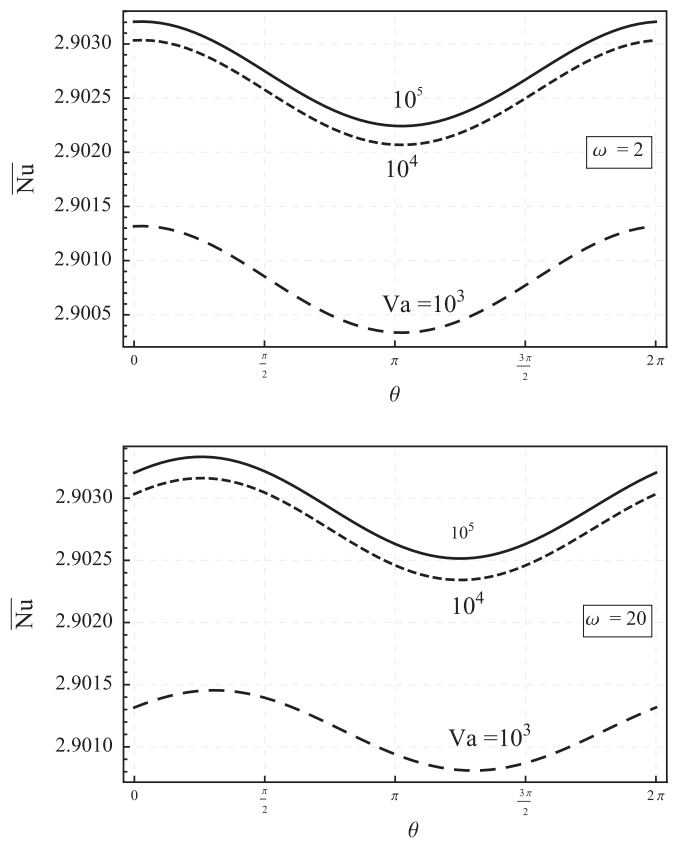

account for the possible importance of time variation of velocity due to imposed time-periodic boundary temperature. It is one of the intentions of the paper to find out if the local acceleration term can be neglected in the modulated Bénard-Darcy convection problem as done in the case of the unmodulated problem.

A linear stability analysis of Bénard-Brinkman convection has been performed by Malashetty and Basavaraja [16] to find the effect of thermal modulation on the onset of convection using Venezian [33] approach. In the present paper a local non-linear stability analysis is done using the Ginzburg-Landau equation for stationary convection. The Nusselt number is calculated by solving the amplitude equation numerically for different values of Vadasz number, thermo-mechanical anisotropy parameters, amplitude and frequency of modulation.

As mentioned earlier, the temperature modulated BénardDarcy problem is approached using the linear matrix differential operator theory. To make the problem analytically tractable small amplitude temperature modulation that facilitates the use of a regular perturbation expansion for the perturbed velocity and temperature has been assumed. Another reason for choosing small amplitude temperature modulation is to discount possible oscillatory convection that might be triggered by large amplitude temperature modulation. As is the case with perturbation procedures, the time-periodic temperature modulation effect arises as an inhomogeneity in a higher order equation with the lowest order equation being homogeneous. The eigenfunction solution of the lowest order problem comes handy in obtaining the solution of the second order system. For the purpose of obtaining the amplitude equation it suffices to know under what condition the third order solution exists. In a functional analytic setting this would impose orthogonality condition between the inhomogeneity in the second-order equation and the solution of the adjoint of the zeroth order problem. Such a solvability condition is known as Fredholm alternative condition and invoking such a condition in our problem resulted in the Ginzburg-Landau equation. Before proceeding to make a discussion of the results it is to be observed that effectively porous medium scales down the Rayleigh number and scales up the Prandtl number. This aspect is incorporated in the paper through the use of Rayleigh-Darcy number and
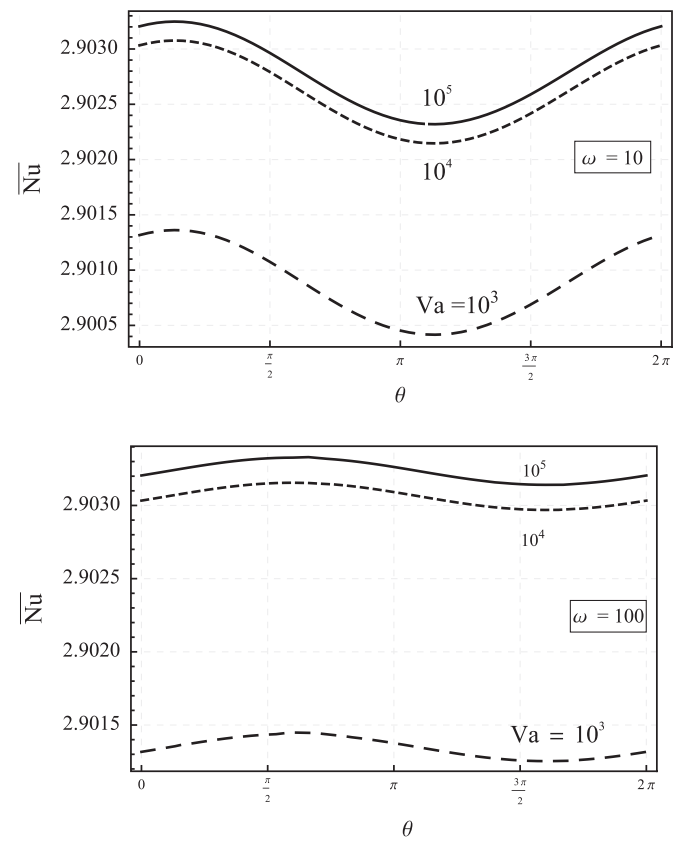

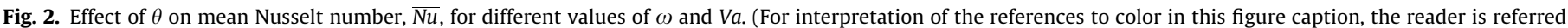
to the web version of this article.) 

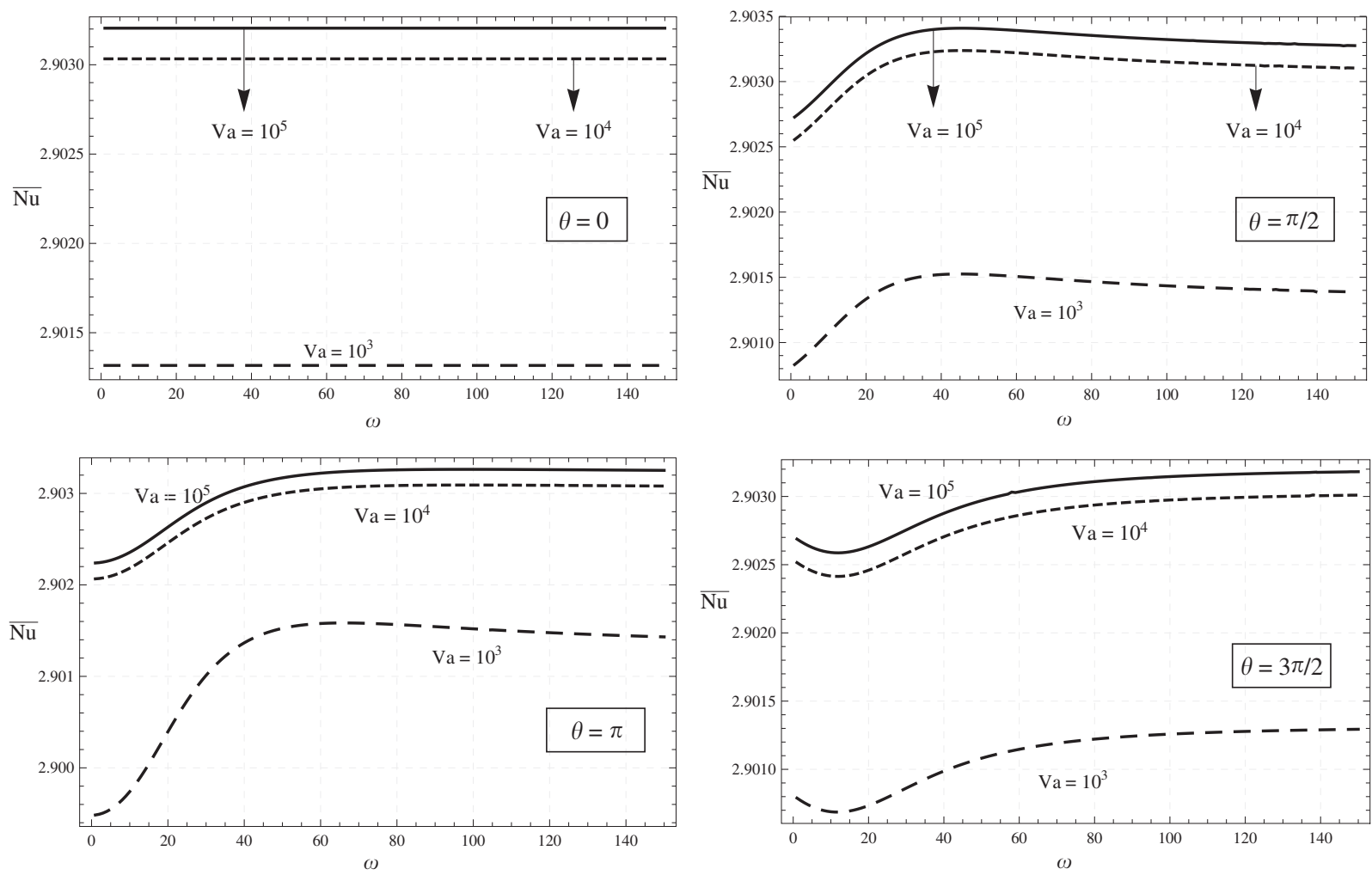

Fig. 3. Effect of $\omega$ on $\overline{N u}$, for different values of $\theta$ and $V a$.

Table 1

Limiting cases of the present study.

\begin{tabular}{|c|c|c|c|c|}
\hline$\alpha_{1}^{2}$ & $\alpha_{2}^{2}$ & Va (Type of convection) & Authors & $\mathbf{P}=\frac{1}{\alpha_{2}^{2}}\left(1+\frac{\alpha^{2} \alpha_{2}^{2}}{\alpha_{1}^{2} \mathbf{V a}}\right) a$ \\
\hline$\alpha^{2}$ & $\alpha^{2}$ & Va (Bénard-Darcy) & Lapwood [15] & $\frac{1}{\alpha^{2}}\left(1+\frac{\alpha^{2}}{V a}\right)$ \\
\hline$\alpha^{2}$ & $\alpha^{2}$ & $\alpha^{2} \operatorname{Pr}$ (Bénard-Rayleigh) & Rayleigh [22] & $\frac{1}{\alpha^{2}}\left(1+\frac{1}{\operatorname{Pr}}\right)$ \\
\hline$\alpha^{2}$ & $\alpha^{2}$ & $\left(\alpha^{2} P r+V a\right)$ (Bénard-Brinkman) & Nield and Bejan [17] & $\frac{1}{\alpha^{2}}\left(1+\frac{\alpha^{2}}{\left(\alpha^{2} P r+V a\right)}\right)$ \\
\hline$\alpha^{2}$ & $\alpha^{2}$ & $\left(\alpha^{2}+Q\right) \operatorname{Pr}$ (Bénard-Chandrasekhar) & Siddheshwar and Pranesh [30] & $\frac{1}{\alpha^{2}}\left(1+\frac{\alpha^{2}}{\operatorname{Pr}\left(\alpha^{2}+Q\right)}\right) b$ \\
\hline
\end{tabular}

${ }^{\text {a }} \mathbf{P}$ is the coefficient of $d \mathcal{A} / d \tau$ in (32).

${ }^{\mathrm{b}} \mathrm{Q}$ is the Chandrasekhar number [28].

Prandtl-Darcy number (or Vadasz number). In effect, it also means that porous media dampens time-variations of velocity. Since the porous layer is assumed to be densely packed, moderate and/or large values of Vadasz number are considered.

The results on heat transport as depicted in Fig. 2 are new. The plot 2 has been included to highlight the fact that the effect of modulation on mean Nusselt number depends crucially on both the phase-difference and frequency rather than on only the choice of the frequency of small-amplitude modulation. From the Fig. 2 and 3 it is evident that for a given frequency of modulation there is a range of $\theta$ in which $\overline{N u}$ increases with increase in $\theta$ and another range in which $\overline{N u}$ decreases. Thus, the inference that can be drawn from this is that combination of choices of $\omega$ and $\theta$ can be made depending on the demands on heat transport in an application situation. Heat transfer can be regulated (enhanced or reduced) with the external mechanism of temperature modulation. The only thing to be borne in mind in the case of the temperature modulation is that the boundary temperatures should not be synchronized. It is to be noted here that the results in Fig. 2 are compatible with the following results on Rayleigh number reported by Malashetty and Basavaraja [16]. On choosing a different set of parameters' values, especially $\theta$, we noticed that the following can also be true

$\left[R a_{c}\right]_{\delta=0}>\left[R a_{c}\right]_{\delta \neq 0}$,

in the case of asymmetric temperature modulation.

Fig. 3 is a conventional Venezian-type plot of $\overline{N u}$ versus $\omega$. It basically reiterates what is said in the context of Fig. 2 and further the results in the figure are compatible with those reported by Malashetty and Basavaraja [16]. Another useful result 
discernible from Fig. 2 and 3 is that $\overline{N u}$ becomes independent of $\mathrm{Va}$ (considering a three digit accuracy) for $\mathrm{Va}>10^{4}$.

Using the results of the present study, certain qualitatively similar results can be obtained for other problems such as Bénard-Rayleigh, Bénard-Brinkman and Bénard-Chandrasekhar convection by replacing $\alpha_{1}^{2}, \alpha_{2}^{2}$ and $D a^{-1}$ in the expression of $R a_{c}^{(0)}$ (Eq. (26)) and in the amplitude equation (Eq. (32)) with appropriate quantities. Table 1 spells out clearly as to how the results of the above three problems may be extracted from those of the paper.

\section{Conclusions}

Weakly non-linear stability analyses pursued in the paper reveals new results on onset as well as on heat transport. The following conclusions may be summarized from the figures documented in the paper:

1. $[\overline{N u}]_{\delta=0} \approx[\overline{N u}]_{\delta \neq 0}$ for synchronous temperature modulation. This result suggests that to regulate heat transport synchronous temperature modulation is not suitable.

2. $[\overline{N u}]_{\delta=0}>[\overline{N u}]_{\delta \neq 0}$ or $[\overline{N u}]_{\delta=0}<[\overline{N u}]_{\delta \neq 0}$ for asynchronous temperature modulation, depending on the choice of $\omega$ and $\theta$.

3. Computations reveal the following result on the effect of $\xi$ and $\eta$ on $R a_{c}$ and $\overline{N u}$ for all values of $\omega$ and $\theta$ :

$$
\begin{aligned}
& \text { (a) }\left[R a_{c}\right]_{\xi=1}>\left[R a_{c}\right]_{\xi<1} {[\overline{N u}]_{\xi=1}<[\overline{N u}]_{\xi<1} } \\
& \text { (b) }\left[R a_{c}\right]_{\eta=1}<\left[R a_{c}\right]_{\eta<1} \\
& {[\overline{N u}]_{\eta=1}>[\overline{N u}]_{\eta<1} }
\end{aligned}
$$

These conform to reported results of earlier works pertaining to temperature/gravity modulated Bénard-Darcy convection $[27,28]$.

4. $\overline{N u}$ increases with increase in $V a$, for all values of $\omega$ and $\theta$.

5. The inclusion of the local acceleration term is significant only in the case when $V a<10^{4}$. This is true for both modulated and unmodulated problems. The importance of this term is more when the frequency of modulation is not so small.

6. Qualitatively, there are similarities between the parameters' influence on heat transport in modulated and unmodulated systems. This again is based on an observation of the results of computation carried out in the paper.

7. The results of Bénard-Rayleigh, Bénard-Brinkman and BénardChandrasekhar convection problems with temperature modulation can be recovered from the present study that uses lower order equations than the ones used in the above three problems.

\section{Acknowledgments}

Author OPS is grateful to the University Grants Commission, Government of India, for providing him financial assistance in the form of Dr. D. S. Kothari Post-doctoral Fellowship. The authors are grateful to the referees for their most useful comments that helped refine the paper to the present form.

\section{References}

[1] G. Ahlers, P.C. Hohenberg, M. Lucke, Thermal convection under external modulation of the driving force, I. The Lorenz model, Physical Review A 32 (6) (1985) 3493-3518.
[2] B.S. Bhadauria, Thermal modulation of Rayleigh-Bénard convection in a sparsely packed porous medium, Journal of Porous Media 10 (2) (2007) 175-188.

[3] B.S. Bhadauria, O.P. Suthar, Effect of thermal modulation on the onset of centrifugally driven convection in a vertical rotating porous layer placed far away from the axis of rotation, Journal of Porous Media 12 (3) (2009) 239-252.

[4] B.S. Bhadauria, P.K. Bhatia, L. Debnath, Weakly non-linear analysis of Rayleigh-Benard convection with time periodic heating, International Journal of Non-Linear Mechanics 44 (2009) 58-65.

[5] B.S. Bhadauria, P.K. Bhatia, Time-periodic heating of Rayleigh-Bénard convection, Physica Scripta 66 (1) (2002) 59-65.

[6] B.S. Bhadauria, P.G. Siddheshwar, O.P. Suthar, Non-linear thermal instability in a rotating viscous fluid layer under temperature/gravity modulation, ASME, Journal of Heat Transfer 134 (2012) 102502-1-102502-9.

[7] S. Chandrasekhar, Hydrodynamic and Hydromagnetic Stability, Oxford University Press, London, 1961.

[8] B. Chhuon, J.P. Caltagirone, Stability of a horizontal porous layer with timewise periodic boundary conditions, ASME Journal of Heat Transfer 101 (1979) 244-248.

[9] S.H. Davis, The stability of time periodic flows, Annual Review of Fluid Mechanics 8 (1976) 57-74.

[10] J.F. Epherre, Critére d'apparition de la convection naturalle dans une couche poreuse anisotrope, Revue Générale de Thermique 168 (1975) 949-950.

[11] A.V. Getling, Rayleigh-Benard Convection: Structures and Dynamics, World Scientific Press, Singapore, 2001.

[12] S. Govender, Stability of convection in a gravity modulated porous layer heated from below, Transport in Porous Media 57 (1) (2004) 113-123.

[13] G.M. Homsy, Global stability of time-dependent flows. Part 2. Modulated fluid layers, Journal of Fluid Mechanics 62 (1974) 387-403.

[14] D.B. Ingham, I. Pop (Eds.), Transport Phenomenon in Porous Media, II, Pergamon, 2002.

[15] E.R. Lapwood, Convective of a fluid in a porous medium, Proceedings of the Cambridge Philosophical Society 44 (1948) 508-521.

[16] M.S. Malashetty, D. Basavaraja, Rayleigh-Bénard convection subject to time dependent wall temperature/gravity in a fluid-saturated porous medium, Heat and Mass Transfer 38 (2002) 551-563.

[17] D.A. Nield, A. Bejan, Convection in Porous Media, Springer-Verlag, New York, 2006.

[18] J.J. Niemela, R.J. Donnelly, External modulation of Rayleigh-Bénard convection, Physical Review Letters 59 (1987) 2431-2434.

[19] J.K. Platten, J.C. Legros, Convection in Liquids, Springer-Verlag, Berlin, 1984.

[20] K.R. Rajagopal, G. Saccomandi, L. Vergori, A systematic approximation for the equations governing convection-diffusion in a porous medium, Nonlinear Analysis: Real World Applications 11 (4) (2010) 2366-2375.

[21] V.R.K. Raju, S.N. Bhattacharya, Onset of thermal instability in a horizontal layer of fluid with modulated boundary temperatures, Journal of Engineering Mathematics 66 (2010) 343-351.

[22] S. Rayleigh, On convective currents in a horizontal layer of fluid when the higher temperature is on the under side, Philosophical Magazine 32 (1916) 529-546.

[23] M.H. Roppo, S.H. Davis, S. Rosenblat, Bénard convection with time-periodic heating, Physics of Fluids 27 (4) (1974) 796-803.

[24] S. Rosenblat, G.A. Tanaka, Modulation of thermal convection instability, Physics of Fluids 14 (7) (1971) 1319-1322.

[25] S. Rosenblat, D.M. Herbert, Low frequency modulation of thermal instability, Physics of Fluids 14 (1971) 1319-1322.

[26] P.G. Siddheshwar, A series solution for the Ginzburg-Landau equation with a time-periodic coefficient, Applied Mathematics 1 (6) (2010) 542-554.

[27] P.G. Siddheshwar, R.K. Vanishree, A.C. Melson, Study of heat transport in Bénard-Darcy convection with g-jitter and thermomechanical anisotropy in variable viscosity liquids, Transport in Porous Media 92 (2) (2012) 277-288.

[28] P.G. Siddheshwar, B.S. Bhadauria, A.K. Srivastava, An analytical study of nonlinear double-diffusive convection in a porous medium under temperature/gravity modulation, Transport in Porous Media 91 (2012) 585-604.

[29] P.G. Siddheshwar, B.S. Bhadauria, P. Mishra, A.K. Srivastava, Study of heat transport by stationary magneto-convection in a Newtonian liquid under temperature or gravity modulation using Ginzburg-Landau model, International Journal of Non-Linear Mechanics 47 (5) (2012) 418-425.

[30] P.G. Siddheshwar, S. Pranesh, Effect of temperature/gravity modulation on the onset of magneto-convection in electrically conducting fluids with internal angular momentum, Journal of Magnetism and Magnetic Materials 219 (2) (2000) 153-162.

[31] B. Straughan, The Energy Method, Stability, and Nonlinear Convection, Applied Mathematical Science Series, second ed., Springer-Verlag, New York, 2004.

[32] K. Vafai, Handbook of Porous Media, Taylor and Francis, New York, 2005.

[33] G. Venezian, Effect of modulation on the onset of thermal convection, Journal of Fluid Mechanics 35 (1969) 243-254. 